\title{
Tumores gástricos peculiares: tumor glómico, schwannoma, leiomioma y cistoadenoma en el páncreas ectópico. Serie de casos y revisión de la literatura
}

\author{
Unusual gastric tumors: Glomus tumor, schwannoma, leiomyoma, and \\ cystadenoma in ectopic pancreas. A case report and literature review
}

Cristina Padilla-Herrera, ${ }^{*}$ (D) Liliana María Suárez, ${ }^{2}$ (D) Raúl Enrique Guevara, ${ }^{2}$ (D) Juan Carlos Barriga. ${ }^{2}$ (C)

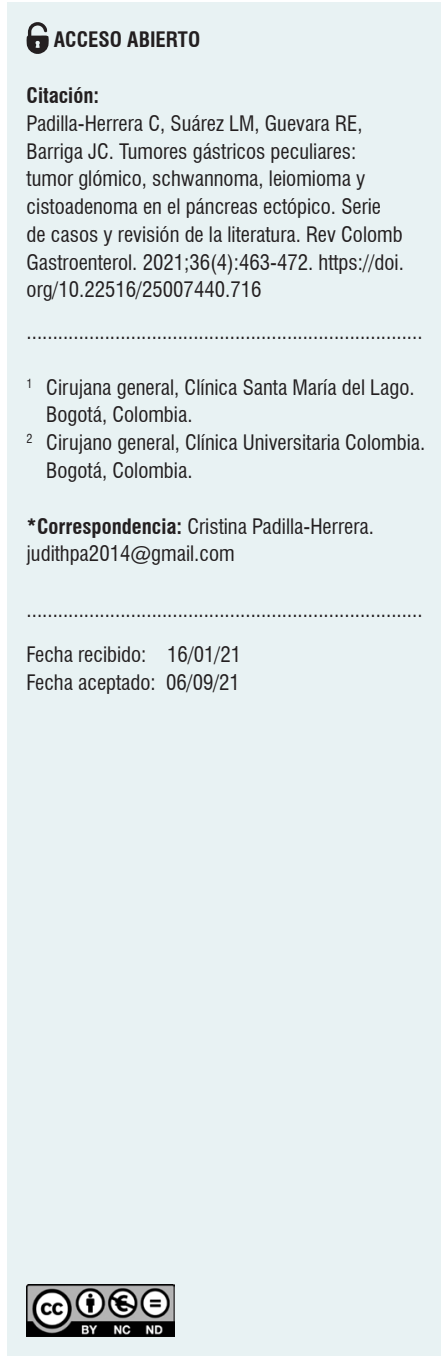

\begin{abstract}
Resumen
Introducción: la patología gástrica es excepcionalmente benigna, se registra a nivel mundial un porcentaje de aparición de las mismas del 0,005 \%-4\%. Además de lo anterior, suele plantearse como primer diagnóstico diferencial un carcinoma gástrico, por lo que la mayoría de los pacientes termina en un procedimiento quirúrgico mayor. El objetivo de este estudio fue describir una serie de casos registrados en una institución de alta complejidad, así como la revisión de la literatura al respecto del tema. Pacientes y métodos: se realizó la revisión de las bases de datos del servicio de cirugía general de la Clínica Universitaria Colombia y se encontraron 4 tumores gástricos de presentación inusual, de los cuales se hizo revisión de la historia clínica y las imágenes disponibles (diagnósticas y de las piezas de cirugía). Resultados: en la revisión de la base de datos, se encontraron 4 casos, 2 de sexo femenino y 2 de sexo masculino, con una media de edad de 51 años (26-75 años). El $100 \%$ de los pacientes tenía una lesión en la región antral y solo uno de ellos tenía una segunda lesión a nivel prepilórico. La totalidad de los pacientes fue llevada a gastrectomía subtotal con reconstrucción en $Y$ de Roux por abordaje laparoscópico y la media de estancia hospitalaria fue de 3,5 días (2-6 dias). Conclusión: para determinar la mejor opción de tratamiento en general en pacientes con lesiones gástricas siempre hay que considerar tanto las características endoscópicas y endosonográficas como las histológicas.
\end{abstract}

\section{Palabras clave}

Neoplasias gástricas, leiomioma, cistoadenoma, tumor glómico.

\begin{abstract}
Introduction: Gastric pathology is exceptionally benign with a percentage of appearance between $0.005 \%$ $-4 \%$ worldwide. Moreover, gastric carcinoma is often suggested as the first differential diagnosis and for that reason, the outcome for a vast majority of admitted patients is a major surgical procedure. This study aims to describe a series of cases recorded in a high complexity medical institution and to carry out a literature review related to that subject. Patients and Methods: Databases from the general surgery service of the Clínica Universitaria Colombia were reviewed, and four unusual gastric tumors were found. A review of the related medical records and available images (diagnostic images and surgical elements) was performed. Results: After the database review, four cases were found: two female cases and two male cases, with a mean age of 51 years (26-75 years). All the patients had lesions in the antrum area and only one of them has an additional lesion in the prepyloric region. All patients were treated with a laparoscopic subtotal gastrectomy with Rouxen- $Y$ reconstruction and the mean length of hospital stay was 3.5 days (2-6 days). Conclusion: It is required to consider the endoscopic, endosonographic, and histological examinations and their features to determine the best treatment for patients with gastric lesions.
\end{abstract}

Keywords

Gastric neoplasia, Leiomyoma, Cystadenoma, Glomus tumor. 


\section{INTRODUCCIÓN}

La patología gástrica oncológica más común es la de origen maligno, que representa la tercera causa de muerte por cáncer a nivel mundial con una incidencia estimada de 950000 casos al año ${ }^{(1)}$. Sin embargo, existe un pequeño porcentaje de pacientes, que representa el 0,005 \%-4\%, que padecen neoplasias gástricas de origen benigno, como es el caso de los schwannomas, leiomiomas y tumores glómicos. La mayoría de estas lesiones suele confundirse con tumores estromales, para lo cual se hace fundamental el estudio de inmunohistoquímica con el fin de registrar la negatividad de estos para la sinaptofisina y la cromogranina $\mathrm{A}^{(2)}$.

Dentro de estas patologías poco frecuentes también se encuentra el páncreas ectópico o localización atípica del tejido pancreático; es decir, fuera de sus límites anatómicos o conexiones vasculares normales; frecuentemente se localiza en el tercio superior del estómago y predominantemente a lo largo de la curvatura mayor. Esta afección suele encontrarse solo en el 0,6\%-13\% de las autopsias clínicas ${ }^{(3)}$.

Estos tumores gástricos comúnmente se encuentran entre la cuarta y la sexta décadas de la vida, y suelen cursar con sintomatología inespecífica, que va desde un dolor abdominal insidioso hasta un cuadro de obstrucción intestinal ${ }^{(1-16)}$.

En la presente publicación se describe la experiencia de un centro de práctica clínica de cuarto nivel en el abordaje diagnóstico y terapéutico de los tumores gástricos inusuales, así como una revisión de la literatura al respecto de cada tipo de tumor.

\section{PACIENTES Y MÉTODOS}

El presente estudio describe las condiciones clínicas y el tratamiento de 4 pacientes que fueron diagnosticados con algún tipo de tumor gástrico benigno, en el período comprendido entre enero de 2012 y abril de 2017. Los casos aquí presentados fueron identificados en las bases de datos del servicio de cirugía general de la Clínica Colombia; posteriormente, se realizó una revisión de historia clínica y de las imágenes disponibles de cada caso en particular.

En cuanto a los pacientes seleccionados, por una parte, se encontró que la totalidad de ellos refería dolor abdominal al momento de la primera valoración médica y que, dentro de los estudios solicitados al inicio, se incluyeron imágenes tomográficas y endoscópicas. Por otra parte, todos los pacientes fueron llevados a junta multidisciplinaria, en la que luego de revisar los estudios se plantearon como principales diagnósticos diferenciales un tumor del estroma gastrointestinal (GIST; por las características en imagen) y un adenocarcinoma gástrico; la decisión en la totalidad de los casos fue la gastrectomía subtotal laparoscópica.

A continuación, se describen con detalle los 4 casos referidos.

\section{PACIENTE 1}

Se trata de un hombre de 35 años que consultó por un cuadro de dolor epigástrico de 6 meses de evolución, con una pobre modulación con analgésicos convencionales e inhibidores de la bomba de protones (IBP), asociado con episodios de lipotimia en varias oportunidades desde el inicio del cuadro y con antecedente de obesidad mórbida y apnea del sueño. Su examen físico estuvo dentro de límites normales. Se le realizó inicialmente una esofagogastroduodenoscopia y una tomografía axial computarizada (TAC), en las cuales se evidenció una lesión de aspecto tumoral de $2 \mathrm{~cm}$ a nivel de la cara posterior del antro (Figura $\mathbf{1}$ ). Posteriormente, se le solicitó biopsia por ultrasonografía endoscópica (USE), en la cual se evidenció una lesión subepitelial dependiente de la capa muscular que podría corresponder a un GIST.

Con dichos hallazgos, el paciente fue llevado a una antrectomía con reconstrucción en Y de Roux por laparoscopia sin vaciamiento ganglionar (pues las características intraoperatorias sugerían más probablemente un GIST) y se describió como hallazgo intraoperatorio la presencia de un tumor de localización antropilórica.

Durante el posoperatorio presentó un cuadro de hemorragia de vías digestivas altas con resolución espontánea y sin evidencia de úlceras, con sangrado activo en la endoscopia de vías digestivas de control; luego de esto, se detalló en la evolución médica una adecuada tolerancia a la vía oral con el posterior egreso sin complicaciones.

$\mathrm{El}$ análisis histopatológico de la pieza quirúrgica mostró una malformación glomangiovenosa (tumor glómico) originada en la muscular propia, positiva para actina del músculo liso y negativa para cromogranina-sinaptofisina y S100 (Figura 2). Sin requerir más intervenciones, el paciente en la actualidad se encuentra asintomático y con una pérdida de $10 \mathrm{~kg}$ de peso desde la intervención, asociada más probablemente con el tipo de intervención quirúrgica realizado.

\section{PACIENTE 2}

Se trata de una mujer de 68 años que consultó por un cuadro de 1 año de dolor en el epigastrio e hipocondrio derecho, asociado con la disminución de la ingesta de alimentos $\mathrm{y}$, consecuentemente, pérdida de aproximadamente $6 \mathrm{~kg}$ en 8 meses. Tenía antecedentes de fibrilación auricular (FA) e hipertensión arterial (HTA), las cuales se encontraban en manejo con metoprolol y warfarina. En el examen físico había sensación de masa en el epigastrio, por lo que se indicó la realización de esofagogastroduodenoscopia, que reportó una lesión de $10 \mathrm{~cm}$ con centro deprimido y ulcerado y con presencia de fibrina. El reporte de patología no 

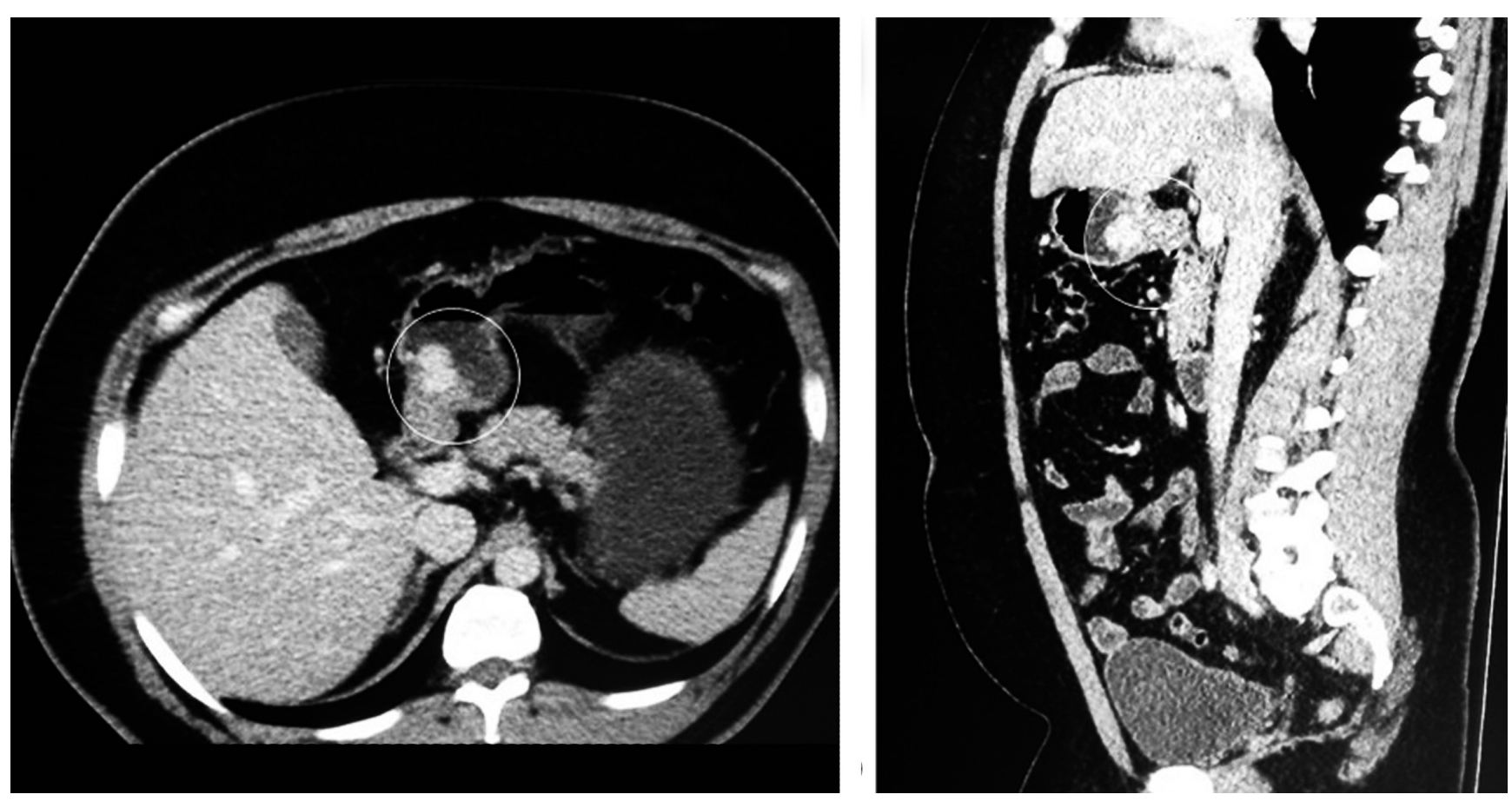

Figura 1. Imagen por TAC en la que se evidencia, en la región antral, la presencia de una lesión sólida exofítica de contornos lobulados que capta el contraste, con un diámetro de 17 × $20 \mathrm{~mm}$, y que podría estar relacionada con un tumor de GIST.

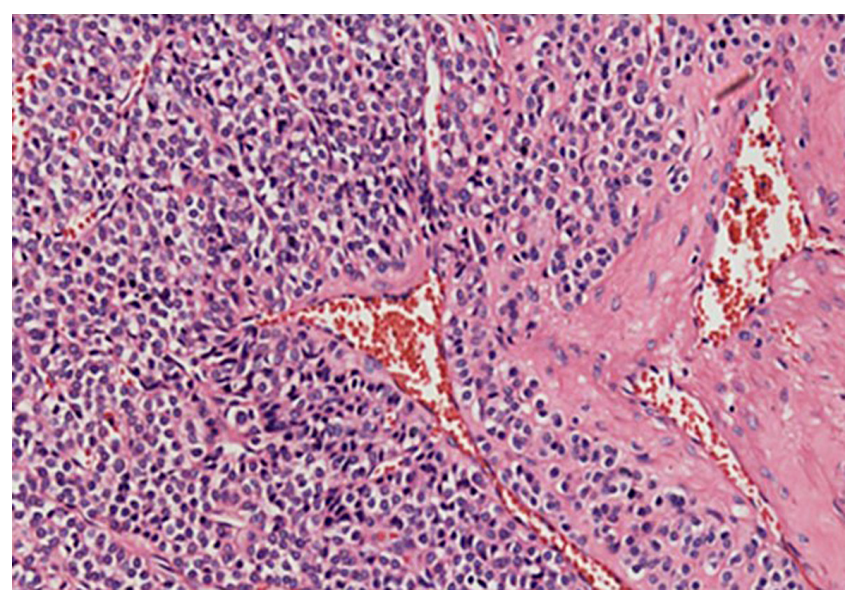

Figura 2. Tumor glómico. Vaso sanguíneo central rodeado por células tumorales homogéneas con núcleo redondo y citoplasma claro. El estudio de inmunohistoquímica con actina de músculo liso mostró positividad fuerte y difusa en las células tumorales (hematoxilina-eosina [H y E] 40x).

fue conclusivo. Adicionalmente, se solicitaron imágenes de extensión, en las que evidenció una gran masa en la curvatura menor del estómago (Figura 3). Con la sospecha de un tumor de GIST, fue llevada a gastrectomía subtotal no radical con reconstrucción en $\mathrm{Y}$ de Roux por laparoscopia.
A nivel intraoperatorio se demostró la presencia de un tumor exofítico en la curvatura menor de aproximadamente $7 \mathrm{~cm}$, pediculado y no adherido a estructuras vecinas. La evolución posoperatoria fue adecuada, por lo que se egresó al cuarto día posoperatorio. El reporte de la patología evidenció un schwannoma con márgenes de sección negativos para compromiso tumoral (Figura 4). Con esta patología fue dada de alta por cirugía general.

\section{PACIENTE 3}

Se trata de un paciente masculino de 75 años que era consumidor crónico de IBP y en los estudios de control en esofagogastroduodenoscopia se evidenció un pólipo en la región antral hacia la curvatura menor que ya se encontraba en seguimiento endoscópico desde 2014 con la toma de una endoscopia más biopsias, todas hasta ese momento negativas para malignidad. En 2016 se evidenció la aparición de una nueva lesión polipoide, por lo que se realizó una USE cuyo resultado demostró una lesión sésil de $8 \mathrm{~mm}$ prepilórica que comprometía hasta la submucosa, y en la pared posterior del antro se encontró un pólipo hiperplásico sésil de $25 \mathrm{~mm}$ con compromiso hasta la submucosa (Figura 5). Durante este estudio se tomaron biopsias que reportaron pólipos hiperplásicos en la curvatura menor sin descartar compromiso tumoral en la base de los pólipos. 

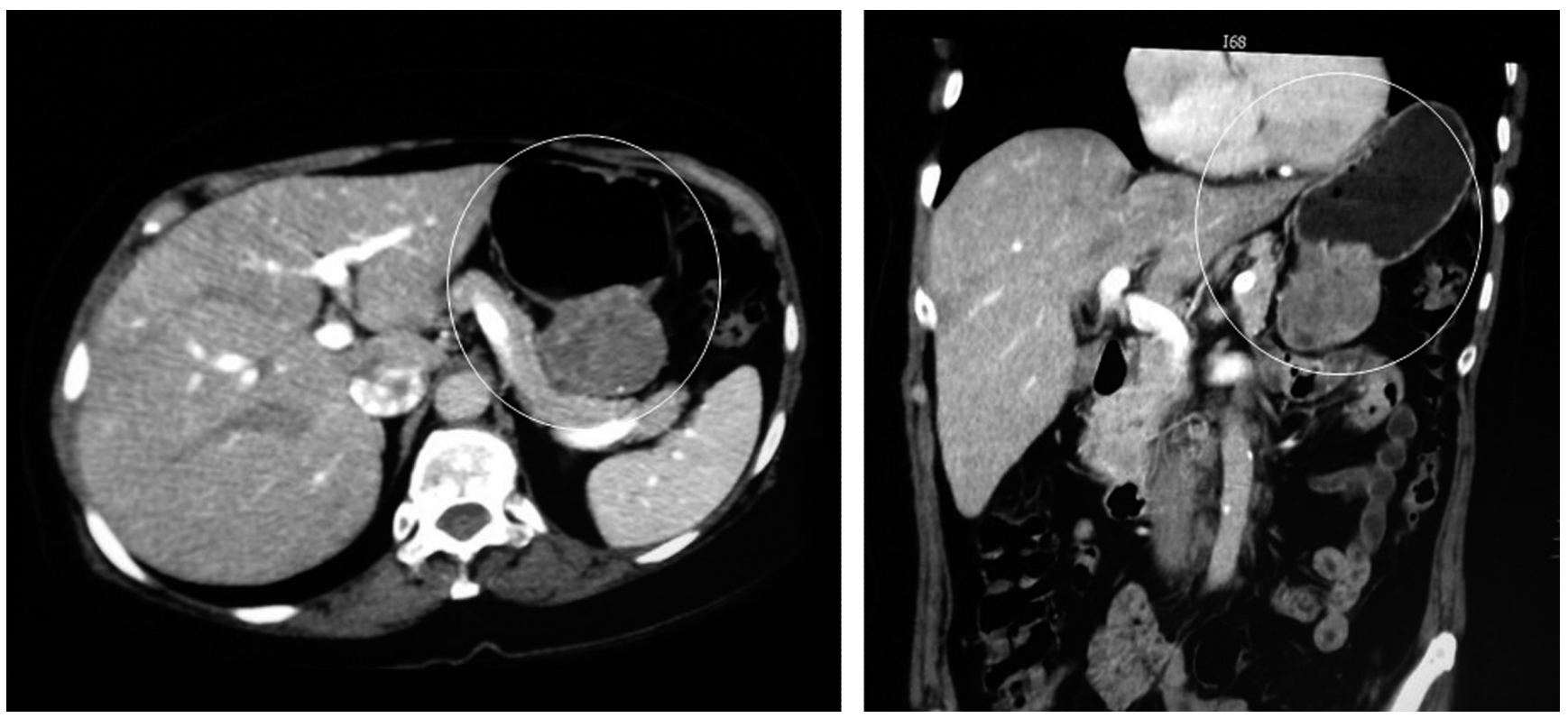

Figura 3. TAC abdominal en la que se evidencia una masa en la curvatura menor del estómago que se proyecta hacia el mesenterio, con una medida aproximada de $50 \mathrm{~mm}$, GIST.
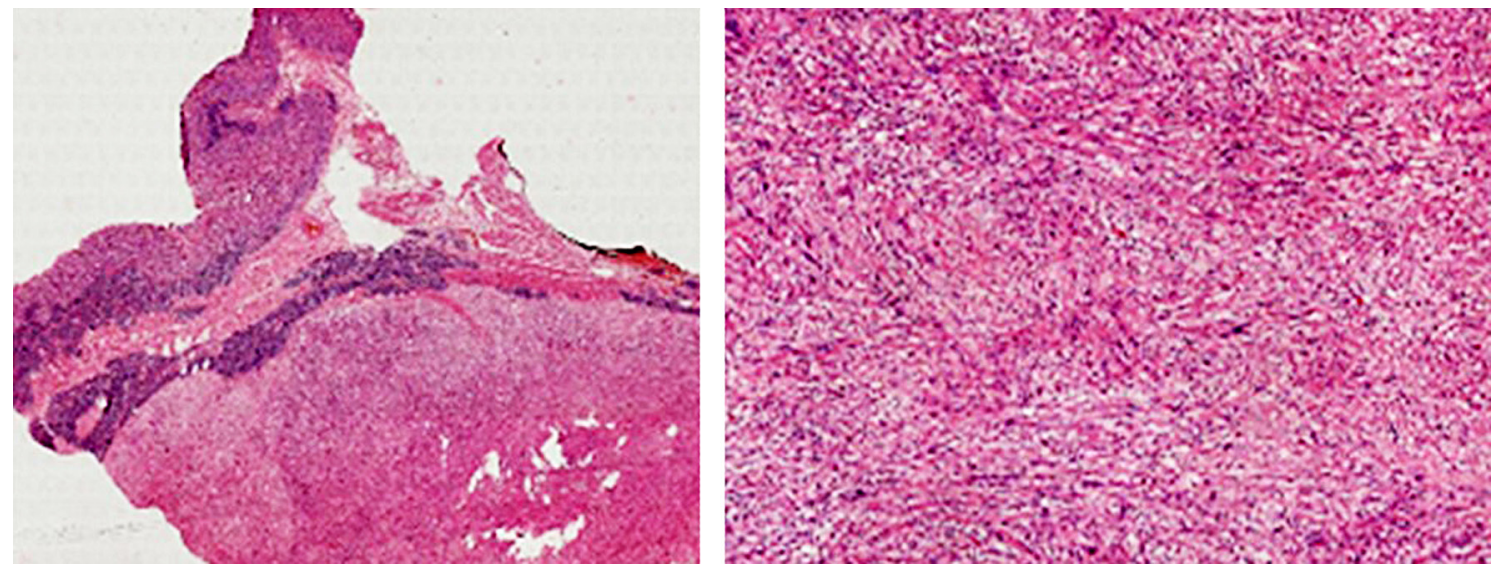

Figura 4. Cuerpo gástrico con tumor benigno que se origina en la muscular. El tumor está constituido por zonas hipercelulares de células fusiformes (Antoni A), alternando con zonas hipocelulares de células ovoides (Antoni B). Las células tumorales presentan reactividad fuerte y difusa para la proteína $S 100$ (H y E 5x y 40x).

Por dicha razón fue llevado a gastrectomía subtotal con vaciamiento ganglionar D2 por laparoscopia, y no se reportaron complicaciones en el posoperatorio.

El paciente fue valorado a los 10 días del posoperatorio, con un análisis histopatológico de la pieza quirúrgica que reportó leiomiomas submucosos en número de dos, sin cambios displásicos o metaplásicos, con bordes de sección libres, tejido fibroadiposo con congestión vascular y tres ganglios linfáticos con hiperplasia sinuhistiocitaria. Dados los hallazgos de la patología, no se le realizaron estudios de inmunohistoquímica. Con dichos resultados el paciente fue dado de alta por la especialidad de cirugía.

\section{PACIENTE 4}

Se trata de una mujer de 26 años que consultó por un cuadro de epigastralgia, con antecedente de dos hospitalizaciones previas por cuadros de hemorragia de vías digestivas altas 

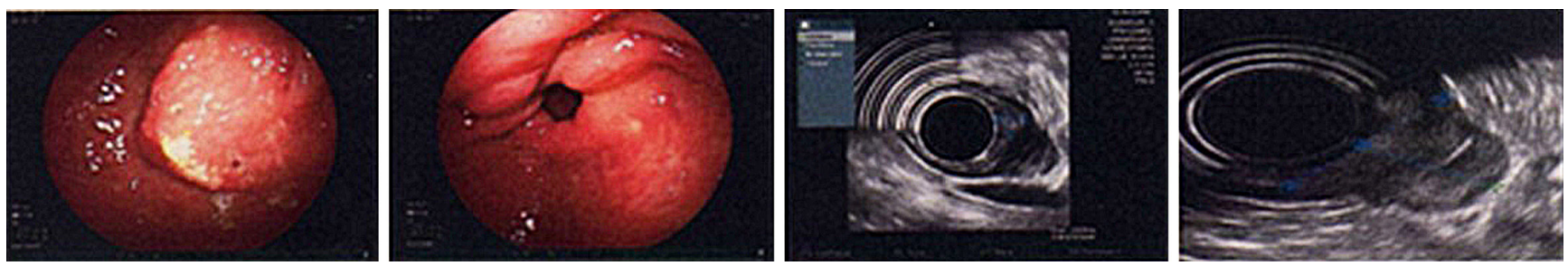

Figura 5. Pólipo hiperplásico sésil en la región prepilórica de $6 \mathrm{~mm}$ x $8 \mathrm{~mm}$ de diámetro que compromete hasta la capa submucosa, respetando la capa muscular propia. Pólipo hiperplásico sésil en el antro, pared posterior de $25 \mathrm{~mm}$ de diámetro que compromete hasta la capa submucosa respetando la capa muscular propia.

y con una esofagogastroduodenoscopia de la última hospitalización que reportaba una lesión de $2,5 \mathrm{~cm}$ en el antro gástrico (con un reporte de biopsia compatible con GIST); además, se solicitaron imágenes tomográficas en la que se describió una lesión quística de $3 \mathrm{~cm}$ sobre la curvatura menor (Figura 6). Se llevó a una antrectomía radical por laparoscopia y en el intraoperatorio se evidenció una lesión prepilórica de $4 \mathrm{~cm}$ con presencia de ganglios de hasta 1 $\mathrm{cm}$ hacia la curvatura menor. Durante el posoperatorio presentó un cuadro de íleo, el cual se resolvió a los pocos días con manejo médico, y posteriormente fue dada de alta.

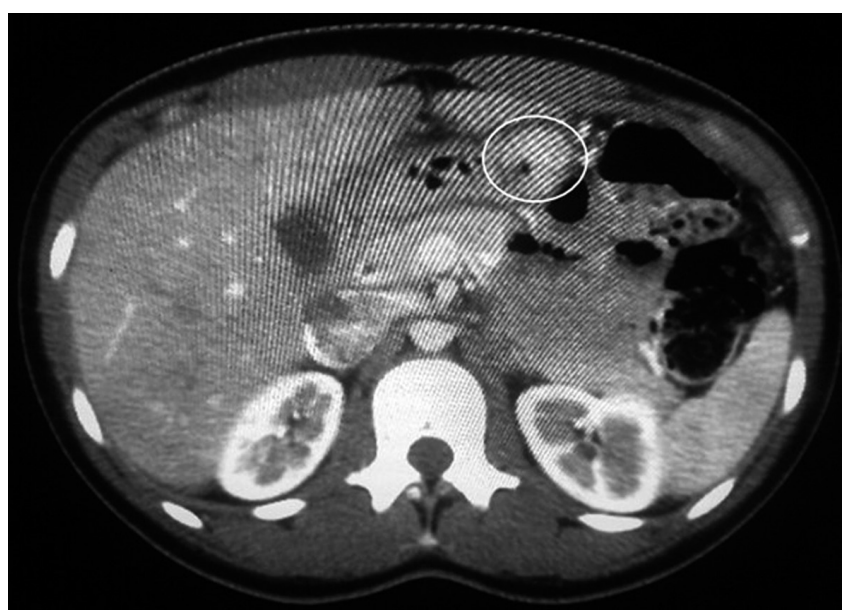

Figura 6. TAC abdominal que reporta una masa quística exofítica multiloculada de paredes y tabiques gruesos e irregulares de $3 \mathrm{~cm}$ al parecer originada en el ligamento gastrohepático, con alteración de la grasa adyacente.

En el control por la consulta externa de cirugía, el reporte de la pieza quirúrgica evidenció un cistoadenoma mucinoso originado en tejido pancreático ectópico (Figura 7). Teniendo en cuenta que la transformación maligna es extremadamente rara, la paciente fue dada de alta por el servicio de cirugía.

\section{RESULTADOS}

De los 4 casos registrados, la mitad era de sexo masculino y la otra mitad de sexo femenino, con una media de edad de 51 años (26-75 años). La clínica predominante fue epigastralgia y solo uno de los casos fue un hallazgo incidental (Tabla 1); el $100 \%$ de los pacientes tenía una lesión en la región antral y solo uno de ellos tenía una segunda lesión a nivel prepilórico. La totalidad de los pacientes fue llevado a gastrectomía subtotal con reconstrucción en $\mathrm{Y}$ de Roux por abordaje laparoscópico, sin complicaciones asociadas ni en el intraoperatorio ni en el posoperatorio. La media de estancia hospitalaria fue de 3,5 días (2-6 días). Todos los pacientes fueron dados de alta por la especialidad.

\section{DISCUSIÓN}

Es muy difícil determinar la etiología de las lesiones gástricas solamente con base en los hallazgos clínicos ${ }^{(2)}$. Además, teniendo en cuenta que las lesiones benignas son poco comunes, es usual que sean confundidas con tumores de origen mesenquimal, como es el caso del GIST, el cual se origina predominantemente en el estómago ${ }^{(4)}$.

En la presente publicación se describen cuatro casos inusuales de tumores gástricos: el primero de ellos es un tumor glómico, patología descrita por primera vez en 1924 por Masson. Es de origen neuromioarterial, está compuesto de células de músculo liso modificadas y se encuentra de más comúnmente en los tejidos blandos de la periferia, como es el caso de las extremidades ${ }^{(5,17)}$.

Los tumores glómicos gástricos son raros y representan tan solo el $1 \%$ de todos los tumores estromales. Hasta hace algunos años solo habían reportados en la literatura 130 casos. Usualmente se localizan de manera predominante en el antro gástrico. Suele presentarse clínicamente con hemorragia digestiva alta y es más común en mujeres entre los 50 y 60 años ${ }^{(6,18)}$. 

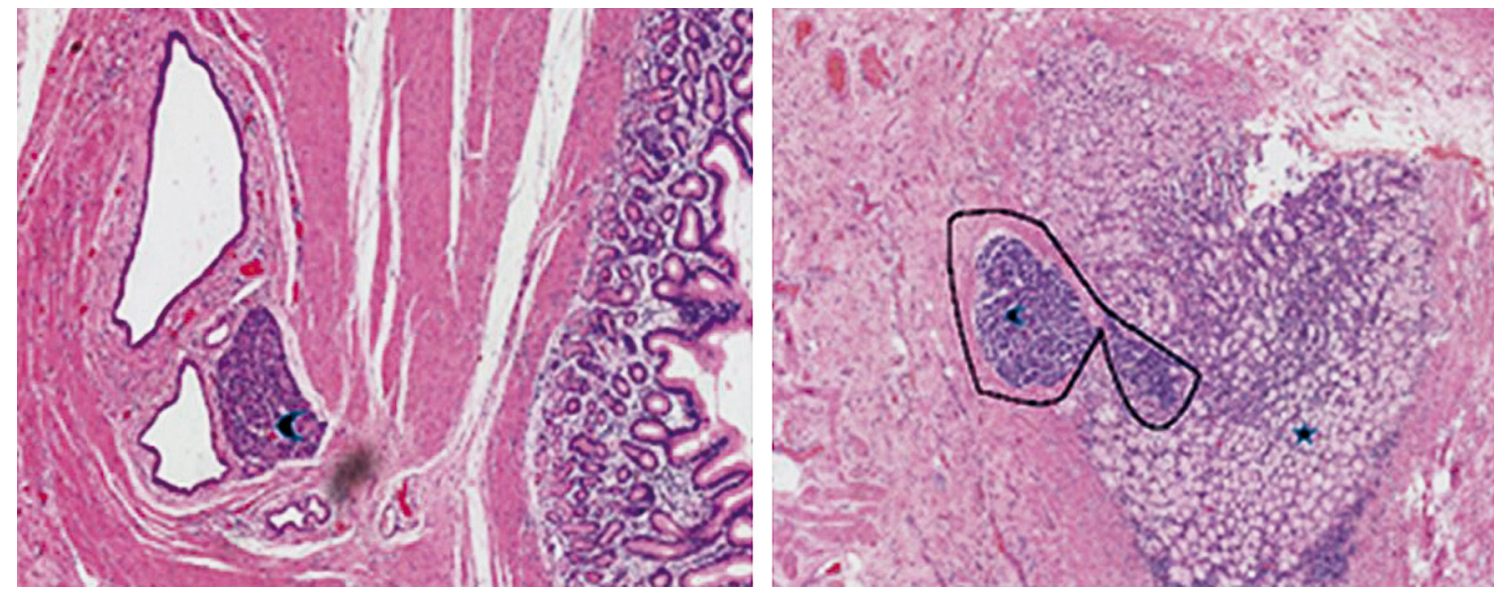

Figura 7. Los hallazgos patológicos del espécimen resecado mostraron un páncreas heterotópico, localizado en lo profundo de la capa muscular del antro, asociado con la presencia de estructuras glandulares y cambios quísticos. El reporte indicó un cistoadenoma mucinoso originado en el tejido pancreático ectópico.

Tabla 1. Características de los pacientes

\begin{tabular}{|c|c|c|c|c|c|c|}
\hline Caso & Sexo & Edad & Cuadro clínico & $\begin{array}{l}\text { Particularidades } \\
\text { endosonográficas }\end{array}$ & $\begin{array}{l}\text { Particularidades } \\
\text { endoscópicas }\end{array}$ & Histopatología \\
\hline $\begin{array}{c}\text { Paciente } \\
1\end{array}$ & Masculino & 35 años & $\begin{array}{l}\text { Epigastralgia + } \\
\text { lipotimia }\end{array}$ & $\begin{array}{l}\text { Lesión subepitelial dependiente } \\
\text { de la capa muscular con un } \\
\text { tamaño aproximado de } 2 \mathrm{~cm}\end{array}$ & & $\begin{array}{l}\text { Tumor glómico actina del } \\
\text { músculo liso (+), negativo para } \\
\text { CD117-CD34, CKAE1/AE3, } \\
\text { cromogranina, sinaptofisina y } \$ 100 \text {. } \\
\text { Ki-67: } 8 \%\end{array}$ \\
\hline $\begin{array}{c}\text { Paciente } \\
2\end{array}$ & Femenino & 68 años & $\begin{array}{l}\text { Epigastralgia } \\
+ \text { pérdida de } \\
\text { peso }\end{array}$ & & $\begin{array}{l}\text { Lesión de } 10 \mathrm{~cm} \text { con } \\
\text { centro deprimido y } \\
\text { ulcerado, y presencia } \\
\text { de fibrina }\end{array}$ & $\begin{array}{l}\text { Schwannoma } \\
\text { S100 (+), desmina (-), CD117(-), } \\
\text { CD34 con positividad focal }\end{array}$ \\
\hline $\begin{array}{l}\text { Paciente } \\
3\end{array}$ & Masculino & 75 años & Asintomático & $\begin{array}{l}\text { Lesión sésil de } 8 \text { mm prepilórica } \\
\text { que compromete hasta la } \\
\text { submucosa y en la pared } \\
\text { posterior del antro, un pólipo } \\
\text { hiperplásico sésil de } 25 \mathrm{~mm} \text { con } \\
\text { compromiso hasta la submucosa }\end{array}$ & & Leiomioma* \\
\hline $\begin{array}{c}\text { Paciente } \\
4\end{array}$ & Femenino & 26 años & $\begin{array}{l}\text { Epigastralgia + } \\
\text { HVDA }\end{array}$ & & $\begin{array}{l}\text { Lesión de } 2,5 \mathrm{~cm} \text { en el } \\
\text { antro gástrico }\end{array}$ & $\begin{array}{l}\text { Cistoadenoma mucinoso en el } \\
\text { páncreas ectópico } \\
\text { Citoqueratina } 7-8 \text { y } 18(+) \text {, antígeno } \\
\text { de membrana epitelial (+) }\end{array}$ \\
\hline
\end{tabular}

*No se le realizó inmunohistoquímica. HVDA: hemorragia de las vías digestivas altas.

Las imágenes por TAC se caracterizan por ser lesiones solitarias hipervascularizadas en la fase arterial y persisten en la fase venosa ${ }^{(19)}$. Como lo describe Chabowski y colaboradores, estos tumores suelen localizarse en la curvatura menor o mayor y en la región prepilórica con un tamaño promedio de $1-4 \mathrm{~cm}$, con una histología que identifica un endotelio vascularizado rodeado de células tumorales con un núcleo no definido y abundante citoplasma; se diferencian de los tumores carcinoides por ser positivos para vimentina y actina del músculo liso, y por ser negativos para cromogranina $^{(7)}$, lo que se correlaciona con los hallazgos histológicos de la pieza quirúrgica de la primera paciente. 
Por su parte, el schwannoma es un raro tumor desarrollado en las células de Schwann cuya localización a nivel gastrointestinal más común suele ser el colon ${ }^{(8)}$. Los de localización gástrica representan el 0,2 \% de las neoplasias del estómago y son el $4 \%$ de todos los tumores benignos gástricos; solo el $8 \%$ de los pacientes presenta como manifestación clínica principal dolor abdominal, mientras que el resto suele debutar con hemorragia de vías digestivas o es un hallazgo incidental en imágenes solicitadas por otra patología ${ }^{(9)}$.

Hasta mayo de 2017 se han reportado solo 221 casos de schwannoma gástrico en la literatura mundial, como lo menciona Bao-Guang $\mathrm{Hu}$ en su publicación, y afecta predominantemente a mujeres entre la quinta y la octava décadas de la vida. Se caracteriza por ser una lesión solitaria localizada predominantemente en el cuerpo del estómago hasta en el 59,3\% de los pacientes, seguido de la región antral en el 26,7\%; y se reporta transformación maligna en solo el $4,5 \%$ de los casos $^{(10)}$.

Estos tumores son proliferaciones de células fusiformes, cuyas agrupaciones se conocen como cuerpos de Verocay. En su interior se encuentran vasos sanguíneos hialinizados y engrosados ${ }^{(20)}$, con infiltrado linfocítico y núcleos con bajo grado de atipia; y las figuras mitóticas son raramente identificables. Suelen ser positivos para S-100 y negativos para actina del músculo liso y desmina, entre otros, que son el estándar de oro para el diagnóstico histopatológico de estos tumores ${ }^{(21)}$.

Las imágenes diagnósticas suelen caracterizarse por ser lesiones bien circunscritas, con mínimos cambios en la fase arterial en la TAC y en la resonancia magnética (RM) usualmente son hiperintensas en $\mathrm{T} 2^{(22)}$.

En el paciente del presente estudio se propuso como diagnóstico diferencial inicial un GIST dada la clínica y los hallazgos endoscópicos, lo que, pese a una biopsia inicial no conclusiva, planteó la necesidad de un manejo quirúrgico no radical (dada la rareza del compromiso linfático en la mayoría de los GIST). Finalmente, la patología final que evidenciaba un tumor que se originaba en la capa muscular propia y se extendía hasta la grasa subserosa fue compatible con un schwannoma.

Hasta 1983 los GIST eran clasificados como tumores de músculo liso e incluían leiomiomas, leiomioblastomas y leiomiosarcomas; y fue hasta el desarrollo de las técnicas de inmunohistoquímica que se demostró que los verdaderos leiomiomas son negativos para CD117 Y CD34, a diferencia de los GIST ${ }^{(11)}$.

En el tercer caso se documentaron de manera incidental unas lesiones que durante el seguimiento endoscópico presentaron aumento de tamaño, razón por la cual se planteó el manejo quirúrgico. El reporte histológico de la pieza quirúrgica fue compatible con un leiomioma de localización corporoantral al cual no se le tomaron estudios de inmunohistoquímica. Los tumores de este tipo, localizados a nivel gástrico, suelen encontrarse frecuentemente a nivel del cardias, y son más comunes en hombres entre los 30 y 35 años de vida. Adicionalmente, Hui Zhu también describió que existen hallazgos tomográficos que permiten diferenciar los tumores estromales de los leiomiomas, pues estos últimos suelen ser de contornos redondeado $u$ ovoide, con patrón de crecimiento intraluminal y ausencia de necrosis intralesional ${ }^{(12)}$.

Histológicamente, son tumores de células fusiformes paucicelulares con baja a moderada celularidad y ligera actividad mitótica. Además, se ha encontrado que la supresión de genes que codifican para las cadenas $a 5$ y a 6 de colágeno tipo IV podría explicar el origen de la formación de estos tumores ${ }^{(23)}$.

Los tumores gástricos se clasifican en tres grandes grupos: los malignos, los benignos y las lesiones que se asemejan a tumores, dentro de las que se encuentra el páncreas ectópico $^{(2)}$. Shultz, en 1727 , fue el primero en describir un páncreas heterotópico, pero no fue sino hasta 1859 que Klob reportó los hallazgos histológicos de este. Por su parte, Heinrich en 1909 clasificó estas lesiones en 3 tipos, de acuerdo con su composición histológica: el primero es un tejido pancreático típico con acinos, ductos e islotes de Langerhans; el segundo contiene acinos y ductos, pero carece de células en islotes; y el tercero, llamado adenomioma, está compuesto de un gran número de ductos. Es frecuente ver músculo liso acompañando la lesión ${ }^{(13,24)}$.

El páncreas ectópico suele encontrarse en el $0,5 \%$ de las laparotomías y el $70 \%$ de los casos es de localización gástrica, principalmente a nivel prepilórico y sobre la curvatura mayor ${ }^{(15)}$. Habitualmente, la ecoendoscopia puede mostrar un crecimiento extramural con irregularidad en los márgenes ${ }^{(16)}$.

A diferencia de los casos registrados en la literatura, el paciente del cuarto caso fue de sexo femenino $y$, al igual que un pequeño porcentaje de los pacientes con esta patología, debutó con sintomatología de dolor abdominal y hemorragia de vías digestivas ${ }^{(2,13-15)}$. Ura y colaboradores reportaron que solo se han registrado en la literatura 7 casos de trasformación maligna de este tipo de lesiones y que la mayoría de ellas estaba localizada en la región antropilórica ${ }^{(16)}$. A pesar de esto y ante la sospecha de malignidad, el manejo debe ser quirúrgico con biopsia por congelación durante el intraoperatorio $^{(13)}$.

Del total de las gastrectomías por laparoscopia realizadas en los últimos años en nuestro centro de práctica, se encontró que un 3,5\% del total correspondía a tumores gástricos benignos, lo que está en relación con los registros encontrados en la literatura mundial, en los que se menciona que más del $95 \%$ de las lesiones gástricas son de tipo adenocarcinoma ${ }^{(25)}$; también se encontró la importancia de los hallazgos en la inmunohistoquímica de la pieza quirúrgica, 
pues estos fueron decisivos en la diferenciación del origen de algunas de las lesiones.

Con base en las recomendaciones descritas en la literatura, se diseñó un algoritmo de abordaje diagnóstico y terapéutico de las lesiones gástricas (Figura 8), en el cual se describe que de preferencia todas las lesiones gástricas sospechosas de malignidad deberían ser llevadas no solamente a endoscopia y endosonografía, (con el fin de determinar características como el grado de profundidad tumoral en la submucosa), sino también a biopsia por ambas vías, y con la información obtenida definir la mejor ruta de manejo ${ }^{(25)}$.

Con lo anterior, las principales lecciones que se pueden deducir son:
- En primer lugar, aunque el cáncer gástrico es la tercera casusa de muerte por cáncer a nivel mundial y siempre debe considerarse dentro del diagnóstico diferencial de las lesiones gástricas, las características de las imágenes preliminares también pueden sugerir fuertemente la presencia de tumores no epiteliales (como los GIST), lo que permitirá plantear las opciones de manejo quirúrgico más convenientes.

- En segundo lugar, aunque los GIST representan un pequeño porcentaje de las lesiones gástricas, usualmente la variante infantil es la que ocasiona metástasis ganglionar; razón por la cual no se plantea el vaciamiento ganglionar para el manejo quirúrgico de estos

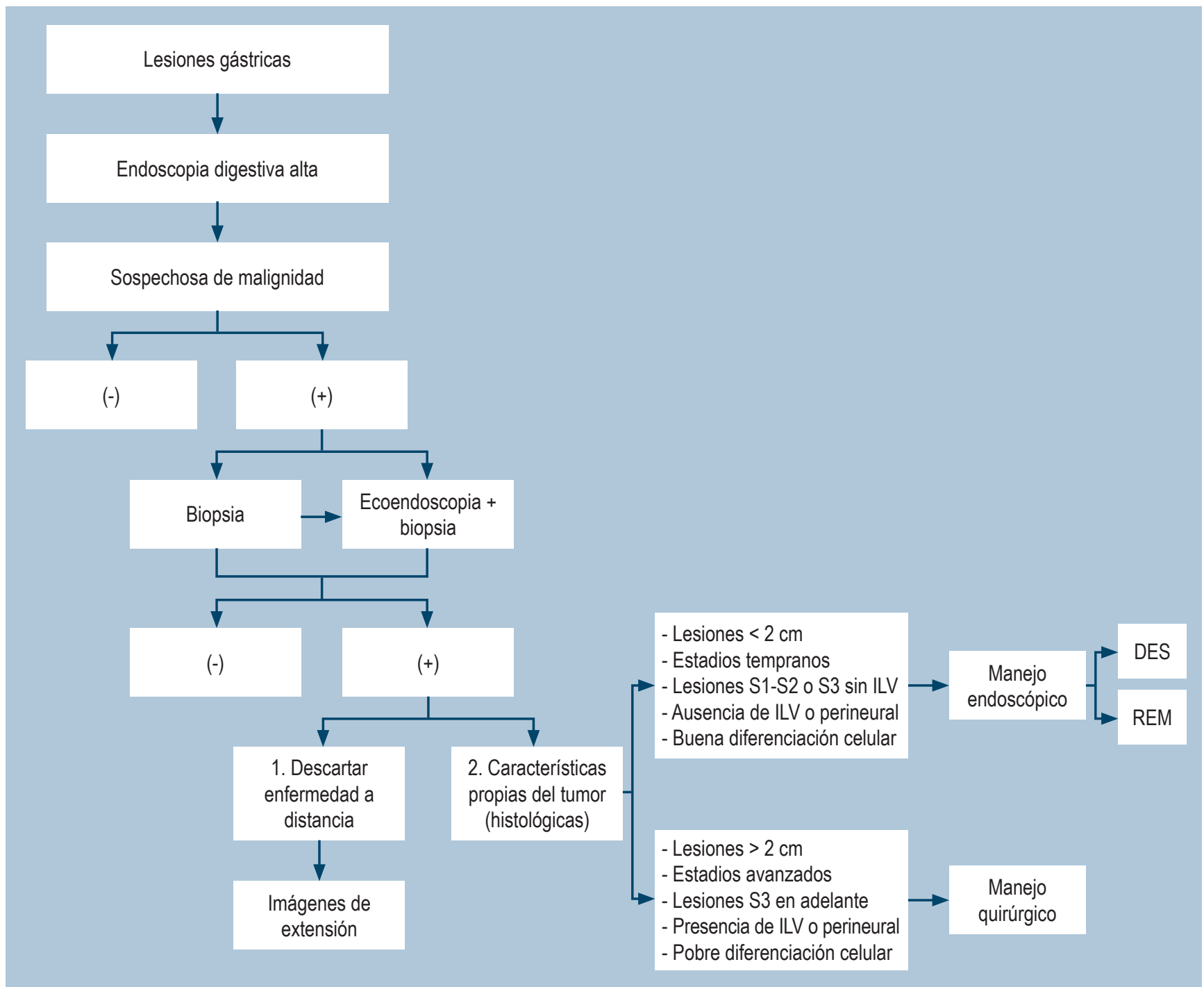

Figura 8. Algoritmo de abordaje diagnóstico y terapéutico de las lesiones gástricas. DES: disección endoscópica de la submucosa; REM: resección endoscópica de la mucosa. 
tumores en el adulto; además, esta fue una de las consideraciones que se tuvo en cuenta al momento de proponer el manejo de los casos arriba descritos.

Así mismo, pese a que los GIST solo requieren manejo con cuña gástrica, por un lado, el hecho de que algunos de los casos aquí reportados demostraran una localización técnicamente demandante para la realización de una cirugía preservadora de órgano (como una cuña gástrica) y que, por otro lado, se considerara la posibilidad de un origen epitelial de las lesiones, se llegó a tomar la determinación de llevar a un manejo quirúrgico más agresivo.

Adicionalmente, en dos de los cuatro casos descritos, dada la alta sospecha de una lesión de origen epitelial (adenocarcinoma), se realizó vaciamiento ganglionar.

- En tercer lugar, usualmente los GIST suelen ser dependientes de la cuarta ecocapa en la endosonografía y suelen ser mayores de $5 \mathrm{~cm}$, razón por la cual, dado el alto potencial de malignidad, siempre se indica en estos casos el manejo quirúrgico como primera opción. Ahora bien, a pesar de que el paciente del primer caso tenía una lesión con un diámetro de $2 \mathrm{~cm}$, los hallazgos de la ecoendoscopia sugerían compromiso de la muscular (cuarta ecocapa), razón por la cual no se consideró el manejo endoscópico inicial. Adicionalmente, y a pesar de que el paciente del tercer caso clínico tenía dos lesiones entre 1 y $2 \mathrm{~cm}$, el reporte de la endosonografía no establecía claramente el compromiso en profundidad de la submucosa; además, las biopsias tomadas no podían descartar compromiso tumoral, por lo que se consideró que se trataba de lesiones altamente sospechosas de malignidad con elevado riesgo de metástasis, razón por la cual también en este paciente se propuso el manejo quirúrgico inicial.

- En cuarto lugar, pese a que las guías internacionales recomiendan la biopsia con aguja fina por endosonografía para lesiones no epiteliales, este procedimiento trae consigo no solo el riesgo de sangrado, sino también de diseminación tumoral intrabdominal o perforación; por estas razones, una vez discutidas las diferentes opciones, en la junta multidisciplinaria se consideró que dadas las particularidades por imagen de las lesiones y el hecho de que en algunos pacientes las biopsias no fueron conclusivas, tal vez era más pertinente llevarlos a un manejo quirúrgico inmediato.

- En quinto lugar, se debe considerar que, según los hallazgos en la patología, en la mayoría de estas lesiones poco frecuentes es necesaria la realización de inmunohistoquímica, la cual permite descartar lesiones del estroma gastrointestinal, entre otras; pese a esto, los estudios de este tipo son muy costosos para realizarlos en pacientes con patologías negativas para malignidad, como fue el caso del paciente 3 .

- Por último, en la evaluación retrospectiva de los casos se considera que, si hubo un sobretratamiento en algunos de ellos, pues tal vez un segundo set de biopsias por ecoendoscopia habría modificado la conducta quirúrgica en aproximadamente la mitad de los casos.

Finalmente, llama la atención que la mayoría de las lesiones atípicas descritas en esta investigación suele ser de localización en el tercio distal del estómago, lo que tal vez pueda sugerir que el origen de la mayoría de ellas podría estar relacionado con los niveles de producción ácida gástrica.

No se describen limitaciones en la realización del presente estudio.

\section{Agradecimientos}

Agradecemos a el servicio de patología de la Clínica Universitaria Colombia, por suministrar las fotografías de las láminas de histología presentadas en esta publicación.

\section{Fuente de financiación}

La presente publicación no ha recibido ayudas específicas provenientes de agencias del sector público, sector comercial o entidades sin ánimo de lucro.

\section{REFERENCIAS}

1. Strand MS, Lockhart AC, Fields RC. Genetics of Gastric Cancer. Surg Clin North Am. 2017;97(2):345-370. https://doi.org/10.1016/j.suc.2016.11.009

2. Lin YM, Chiu NC, Li AF, Liu CA, Chou YH, Chiou YY. Unusual gastric tumors and tumor-like lesions: Radiological with pathological correlation and literature review. World J Gastroenterol. 2017;23(14):2493-2504. https://doi.org/10.3748/wjg.v23.i14.2493
3. Ura H, Denno R, Hirata K, Saeki A, Hirata K, Natori H. Carcinoma arising from ectopic pancreas in the stomach: endosonographic detection of malignant change. J Clin Ultrasound. 1998;26(5):265-8. https://doi.org/10.1002/(sici)10970096(199806)26:5<265::aid-jcu7>3.0.co;2-a

4. Keung EZ, Raut CP. Management of Gastrointestinal Stromal Tumors. Surg Clin North Am. 2017;97(2):437-452. https://doi.org/10.1016/j.suc.2016.12.001 
5. Allan WS, Miller RW. Glomus tumour of the stomach. Br J Surg. 1960;48:145-8.

https://doi.org/10.1002/bjs.18004820810

6. Devanathan M, Jamuna Rani S, Chandramohan SM, Anbarasu S. A rare submucosal tumour of stomach-glomus tumour: A case report. Int J Surg Case Rep. 2015; 1 1:64-67. https://doi.org/10.1016/j.ijscr.2015.04.014

7. Chabowski M, Paszkowski A, Skotarczak J, Dorobisz T, Leśniak M, Janczak D, Janczak D. Glomus Tumor of the Stomach - A Case Report and A Literature Review. Pol Przegl Chir. 2016;88(6):356-358. https://doi.org/10.1515/pjs-2016-0076

8. Kang JH, Kim SH, Kim YH, Rha SE, Hur BY, Han JK. CT Features of Colorectal Schwannomas: Differentiation from Gastrointestinal Stromal Tumors. PLoS One. 2016;11(12):e0166377. https://doi.org/10.1371/journal.pone.0166377

9. Rosero Cuesta D, Gomez Gonzalez C, Pac Sa J, Alastuey Aísa M, Saiz Camín M. Schwannoma gástrico: presentación de dos casos y revisión de la bibliografía. Rev Latinoamericana de Patología. 2013;51(4):227-31.

10. Hu BG, Wu FJ, Zhu J, Li XM, Li YM, Feng Y, Li HS. Gastric Schwannoma: A Tumor Must Be Included in Differential Diagnoses of Gastric Submucosal Tumors. Case Rep Gastrointest Med. 2017;2017:9615359. https://doi.org/10.1155/2017/9615359

11. Lee MJ, Lim JS, Kwon JE, Kim H, Hyung WJ, Park MS, Kim MJ, Kim KW. Gastric true leiomyoma: computed tomographic findings and pathological correlation. J Comput Assist Tomogr. 2007;31(2):204-8. https://doi.org/10.1097/01.rct.0000237812.95875.bd

12. Zhu H, Chen H, Zhang S, Peng W. Differentiation of gastric true leiomyoma from gastric stromal tumor based on biphasic contrast-enhanced computed tomographic findings. J Comput Assist Tomogr. 2014;38(2):228-34. https://doi.org/10.1097/RCT.0b013e3182ab0934

13. Inoue $Y$, Hayashi M, Arisaka Y, Higuchi K, Egashira Y, Tanigawa N. Adenocarcinoma arising in a heterotopic pancreas (Heinrich type III): a case report. J Med Case Rep. 2010;4:39. https://doi.org/10.1186/1752-1947-4-39

14. Bethel CA, Luquette MH, Besner GE. Cystic degeneration of heterotopic pancreas. Pediatr Surg Int. 1998;13(56):428-30. https://doi.org/10.1007/s003830050358

15. Jeong HY, Yang HW, Seo SW, Seong JK, Na BK, Lee BS, Song GS, Park HS, Lee HY. Adenocarcinoma arising from an ectopic pancreas in the stomach. Endoscopy.
2002;34(12):1014-7.

https://doi.org/10.1055/s-2002-35836

16. Ura H, Denno R, Hirata K, Saeki A, Hirata K, Natori H. Carcinoma arising from ectopic pancreas in the stomach: endosonographic detection of malignant change. J Clin Ultrasound. 1998;26(5):265-8.

https://doi.org/10.1002/(sici)10970096(199806)26:5<265::aid-jcu7>3.0.co;2-a

17. Miettinen M, Paal E, Lasota J, Sobin LH. Gastrointestinal glomus tumors: a clinicopathologic, immunohistochemical, and molecular genetic study of 32 cases. Am J Surg Pathol. 2002;26(3):301-11. https://doi.org/10.1097/00000478-200203000-00003

18. Zhang Y, Zhou P, Xu M, Chen W, Li Q Ji Y, Yao L. Endoscopic diagnosis and treatment of gastric glomus tumors. Gastrointest Endosc. 2011;73(2):371-5. https://doi.org/10.1016/j.gie.2010.10.023

19. Haque S, Modlin IM, West AB. Multiple glomus tumors of the stomach with intravascular spread. Am J Surg Pathol. 1992;16(3):291-9. https://doi.org/10.1097/00000478-199203000-00010

20. Perez MT, Farkas J, Padron S, Changus JE, Webster EL. Intrasellar and parasellar cellular schwannoma. Ann Diagn Pathol. 2004;8(3):142-50. https://doi.org/10.1016/j.anndiagpath.2004.03.006

21. Rodriguez E, Tellschow S, Steinberg DM, Montgomery E. Cytologic findings of gastric schwannoma: a case report. Diagn Cytopathol. 2014;42(2):177-80. https://doi.org/10.1002/dc.22913

22. Takeda M, Amano Y, Machida T, Kato S, Naito Z, Kumita S. CT, MRI, and PET findings of gastric schwannoma. Jpn J Radiol. 2012;30(7):602-5. https://doi.org/10.1007/s11604-012-0093-4

23. Miettinen M, Lasota J. Gastrointestinal stromal tumors-definition, clinical, histological, immunohistochemical, and molecular genetic features and differential diagnosis. Virchows Arch. 2001;438(1):1-12. https://doi.org/10.1007/s004280000338

24. Accardo M. Pancreatic heterotopia in the gastric antrum: an ultrastructural report of a case. Ultrastruct Pathol. 2006;30(1):75-83. https://doi.org/10.1080/01913120500214267

25. NCCN Clinical practice guidelines in oncology: Gastric cancer. Estados Unidos. National Comprehensive Cancer Network; 2021 [consultado el 11 de septiembre de 2021]. Disponible en: https://www.nccn.org/professionals/physician_gls/pdf/gastric.pdf 